\title{
Nomogram for prediction of level 2 axillary lymph node metastasis in proven level 1 node-positive breast cancer patients
}

\author{
Yanlin Jiang ${ }^{1, *}$, Hong $\mathrm{Xu}^{2, *}$, Hao Zhang ${ }^{3}$, Xunyan $\mathrm{Ou}^{3}$, Zhen $\mathrm{Xu}^{3}$, Liping $\mathrm{Ai}^{3}$, Lisha \\ Sun ${ }^{4}$ and Caigang Liu ${ }^{1}$ \\ ${ }^{1}$ Department of Breast Surgery, Shengjing Hospital of China Medical University, Shenyang, Liaoning Province, 110004, China \\ ${ }^{2}$ Department of Breast Surgery, Cancer Hospital of China Medical University (Liaoning Cancer Hospital \& Institute), Shenyang, \\ Liaoning Province, 110042, China \\ ${ }^{3}$ Department of Breast Disease and Reconstruction Center, Breast Cancer Key Lab of Dalian, the Second Hospital of Dalian \\ Medical University, Dalian, 116027, China \\ ${ }^{4}$ Department of Surgical Oncology, The First Hospital of China Medical University, Shenyang, 110013, China \\ * Share co-first authorship
}

Correspondence to: Caigang Liu, email: angel-s205@163.com

Keywords: breast cancer, level 2 axillary lymph node metastasis, level 1 axillary lymph node metastasis, nomogram Received: July 08, $2017 \quad$ Accepted: August 08, $2017 \quad$ Published: August 22, 2017

Copyright: Jiang et al. This is an open-access article distributed under the terms of the Creative Commons Attribution License 3.0 (CC BY 3.0), which permits unrestricted use, distribution, and reproduction in any medium, provided the original author and source are credited.

\section{ABSTRACT}

Background: The current management of the axilla in level 1 node-positive breast cancer patients is axillary lymph node dissection regardless of the status of the level 2 axillary lymph nodes. The goal of this study was to develop a nomogram predicting the probability of level 2 axillary lymph node metastasis (L-2-ALNM) in patients with level 1 axillary node-positive breast cancer.

Materials and Methods: We reviewed the records of 974 patients with pathologyconfirmed level 1 node-positive breast cancer between 2010 and 2014 at the Liaoning Cancer Hospital and Institute. The patients were randomized 1:1 and divided into a modeling group and a validation group. Clinical and pathological features of the patients were assessed with uni- and multivariate logistic regression. A nomogram based on independent predictors for the L-2-ALNM identified by multivariate logistic regression was constructed.

Results: Independent predictors of L-2-ALNM by the multivariate logistic regression analysis included tumor size, Ki-67 status, histological grade, and number of positive level 1 axillary lymph nodes. The areas under the receiver operating characteristic curve of the modeling set and the validation set were 0.828 and 0.816 , respectively. The false-negative rates of the L-2-ALNM nomogram were $1.82 \%$ and $7.41 \%$ for the predicted probability cut-off points of $<6 \%$ and $<10 \%$, respectively, when applied to the validation group.

Conclusions: Our nomogram could help predict L-2-ALNM in patients with level 1 axillary lymph node metastasis. Patients with a low probability of L-2-ALNM could be spared level 2 axillary lymph node dissection, thereby reducing postoperative morbidity.

\section{INTRODUCTION}

Owing to its increasing incidence, breast cancer has become one of the most common malignancies, accounting for $15 \%$ of all cancer-related deaths [1-3]. Clear axillary lymph node metastasis greatly influences individualized treatment decision making and has become one of the most important prognostic factors in patients with breast cancer [4-6].

For patients clinically diagnosed as having a level 1 axillary lymph node metastasis (L-1-ALNM), the standard surgical procedure includes primary tumor 
resection and axillary lymph node dissection (ALND; including level 1 and 2 axillary lymph nodes), regardless of whether the level 2 axillary lymph node is positive or negative for malignancy. The ACOSOG Z0011 trial [7] demonstrated axillary lymph node dissection could not benefit them compared with sentinel lymph node biopsy for patients with 12 positive sentinel lymph nodes. In other words, extensive negtive axillary lymph node dissection surgery could not decrease local recurrence rate and improve patient survival in breast cancer patients. However, sentinel lymph node biopsy (SLNB) is not a reasonable and reliable treatment, for the patients with preoperatively confirmed lymph node metastasis. Therefore, level 1 axillary lymph node dissection could be a better option for patients with positive level 1 axillary lymph nodes but without L-2-ALNM. Several retrospective studies showed that less negtive axillary lymph node dissection decreased the incidence of short- and long-term postoperative complications such as limited range of shoulder mobility, lymphedema, and upper arm numbness. Thus, omission of L-2ALND provides patients with better quality of life than performing ALND [8-10]. These findings suggest that a nomogram should be established urgently to evaluate the level 2 axillary lymph node status in patients with level 1 node-positive breast cancer and to identify those in whom L-2-ALND can be omitted.

In some studies, a perfect nomogram has been shown to accurately assess the risk of lymph node metastasis and provide a reliable basis for clinicians to make decisions about breast cancer treatment $[11,12]$. Currently, no well-designed nomogram is available to evaluate the probability of L-2-ALNM in patients with level 1 axillary lymph node metastasis. Therefore, our present research was intended to identify possible predictors of L-2-ALNM and to construct a model to calculate the risk of L-2-ALNM in patients with level 1 axillary node-positive breast cancer, which would increase the accuracy of surgical decision making. With accurate nomogram predictions, patients with a low risk of L-2ALNM can avoid undergoing L-2-ALND.

\section{RESULTS}

\section{Patient characteristics}

Our study included 974 patients randomized $1: 1$ into a modeling set $(n=487)$ and a validation set $(n=487)$. Table 1 depicted the clinical and pathological characteristics of the modeling and validation groups. The L-2-ALNM rates of both groups were the same $(27.9 \%$, 136/487). The clinicopathological characteristics of the patients did not differ significantly between the two groups $(P>0.05)$ in our study population. The univariate analysis of the modeling was shown in Table 2.

\section{Predictors of L-2-ALNM}

After the univariate analysis, the following statistically significant $(P<0.05)$ variables were entered in the multivariate model: tumor size, Ki-67 status, HER2 status, histological grade, and number of PL-1-ALN. The multivariate logistic regression analysis revealed that larger tumor size, positive Ki-67 status, higher histological grade, and greater number of PL-1-ALN remarkably correlated with greater risk of L-2-ALNM (Table 3).

\section{Construction of the nomogram}

To evaluate the preoperative probability of L-2ALNM based on the results of the multivariate logistic regression analyses, the following equation was developed: $\ln (p / 1-p)=0.683 \times a+0.552 \times b+0.728 \times$ $c+0.922 \times \mathrm{d} 1+1.506 \times \mathrm{d} 2-5.314$, where $p$ represents the probability of L-2-ALNM, $a$ represents the number of PL-1-ALN ( 1 if 1 positive level 1 axillary lymph node, 2 if 2 positive level 1 axillary lymph nodes, 3 if 3 positive level 1 axillary lymph nodes, 4 if 4 positive level 1 axillary lymph nodes, and 5 if $>5$ positive level 1 axillary lymph nodes), $b$ represents the tumor size ( 1 if T1, 2 if T2, 3 if T3, and 4 if T4), $c$ represents Ki-67 status ( 0 if negative and 1 if positive), $d 1$ represents histological grade 2 ( 0 if grade 2 [G1] or grade 3 [G3], and 1 if grade $[\mathrm{G} 2]), d 2$ represents histological grade 3 ( 0 if $\mathrm{G} 1$ or $\mathrm{G} 2$, and 1 if G3).

A predictive model presented in the form of a nomogram based on the results of the multivariate logistic regression analysis was established (Figure 1). The nomogram consisted of 7 rows. The first row was the point assignment for each variable, and the second to fifth rows were the four variables of the L-2-ALNM nomogram. The scores of the four variables were added to the total score presented on the scale in row 6 , which corresponds to the prediction of the risk of L-2-ALNM in row 7.

\section{Validation of the L-2-ALNM nomogram}

The internal receiver operating characteristic (ROC) curves in the modeling set (Figure 2) and external ROC in the validation set (Figure 3 ) were used to evaluate the nomogram. In the modeling and validation groups, the area under the curve (AUC) were 0.828 (95\% confidence interval [CI]: $0.788-0.868)$ and 0.816 (95\% CI: $0.772-$ $0.859)$, respectively, which indicates a good predictive ability. Figure 4 showed that our bias-corrected curve was close to the ideal curve, which indicated that the predicted probability of L-2-ALNM was consistent with the actual L-2-ALNM risk. The $p$ value in the Hosmer-Lemeshow test was 0.390 , which confirmed a good fit for the model. The false-negative rate of the model was $7.41 \%$ at cutoff points of $<10 \%$ in the validation group (Table 4 ), and 
Table 1: Comparison between modeling group and validation group by clinicopathological characteristics

\begin{tabular}{|c|c|c|c|}
\hline Variables & Modeling No. (\%) & Validation No. (\%) & $P$-value \\
\hline No. of Patients & $487(100 \%)$ & $487(100 \%)$ & \\
\hline L-2-ALNM & & & 1.000 \\
\hline Yes & $136(27.9)$ & $136(27.9)$ & \\
\hline No & $351(72.1)$ & $351(72.1)$ & \\
\hline Age (year) & & & 0.605 \\
\hline$\leq 45$ & $119(24.4)$ & $126(25.9)$ & \\
\hline$>45$ & $368(75.6)$ & $361(74.9)$ & \\
\hline Menopausal status & & & 0.305 \\
\hline Premenopausal & $237(48.7)$ & $253(52.0)$ & \\
\hline Postmenopausal & $250(51.3)$ & $234(48.0)$ & \\
\hline Tumor size & & & 0.991 \\
\hline $\mathrm{T} 1 \leq 2 \mathrm{~cm}$ & $151(31.0)$ & $150(30.8)$ & \\
\hline $2 \mathrm{~cm}<\mathrm{T} 2 \leq 3 \mathrm{~cm}$ & $266(54.6)$ & $265(54.4)$ & \\
\hline $3 \mathrm{~cm}<\mathrm{T} 3 \leq 5 \mathrm{~cm}$ & $65(13.3)$ & $66(13.6)$ & \\
\hline $\mathrm{T} 4>5 \mathrm{~cm}$ & $5(1.0)$ & $6(1.2)$ & \\
\hline ER status & & & 0.937 \\
\hline Negative & $100(20.5)$ & $99(20.3)$ & \\
\hline Positive & $387(79.5)$ & $388(79.7)$ & \\
\hline PR status & & & 0.777 \\
\hline Negative & $138(28.3)$ & $142(29.2)$ & \\
\hline Positive & $349(71.7)$ & $345(70.8)$ & \\
\hline Her-2 status & & & 0.840 \\
\hline Negative & $433(88.9)$ & $431(88.5)$ & \\
\hline Positive & $54(11.1)$ & $56(11.5)$ & \\
\hline Ki-67 status & & & 0.797 \\
\hline$\leq 20$ & $(47.2)$ & $234(48.0)$ & \\
\hline$>20$ & $257(52.8)$ & $253(52.0)$ & \\
\hline No. of PL-1-ALN & & & 1.000 \\
\hline 1 & $187(38.4)$ & $187(38.4)$ & \\
\hline 2 & $103(21.1)$ & $102(20.9)$ & \\
\hline 3 & $54(11.1)$ & $55(11.3)$ & \\
\hline 4 & $43(8.8)$ & $43(8.8)$ & \\
\hline$\geq 5$ & $100(20.5)$ & $100(20.5)$ & \\
\hline Tumor location & & & 0.089 \\
\hline UOQ & $251(51.5)$ & $284(58.3)$ & \\
\hline LOQ & $48(9.9)$ & $47(9.7)$ & \\
\hline LIQ & $36(7.4)$ & $35(7.2)$ & \\
\hline UIQ & $72(14.8)$ & $69(14.2)$ & \\
\hline Central & $80(16.4)$ & $52(10.7)$ & \\
\hline
\end{tabular}

Abbreviations: UOQ, upper outer quadrant; UIQ, upper inner quadrant; LOQ, lower outer quadrant; LIQ, lower inner quadrant. 
Table 2: Univariate analysis analysis of different variables predicting L-2-ALNM of the modeling set

\begin{tabular}{|c|c|c|c|}
\hline Variables & No L-2-ALNM No. (\%) & L-2-ALNM No. (\%) & $P$-value \\
\hline No. of Patients & $351(100 \%)$ & $136(100 \%)$ & \\
\hline Age (year) & & & 0.957 \\
\hline$\leq 45$ & $86(24.5)$ & $33(24.3)$ & \\
\hline$>45$ & $265(75.5)$ & $103(75.7)$ & \\
\hline Menopausal status & & & 0.811 \\
\hline Premenopausal & $172(49.0)$ & $65(47.8)$ & \\
\hline Postmenopausal & $179(51.0)$ & $71(52.2)$ & \\
\hline Tumor size & & & $<0.001$ \\
\hline $\mathrm{T} 1 \leq 2 \mathrm{~cm}$ & $124(35.3)$ & 27 (19.9) & \\
\hline $2 \mathrm{~cm}<\mathrm{T} 2 \leq 3 \mathrm{~cm}$ & $193(55.0)$ & $73(53.7)$ & \\
\hline $3 \mathrm{~cm}<\mathrm{T} 3 \leq 5 \mathrm{~cm}$ & $34(9.7)$ & $31(22.8)$ & \\
\hline $\mathrm{T} 4>5 \mathrm{~cm}$ & $0(0.0)$ & $5(3.7)$ & \\
\hline Histological grade & & & $<0.001$ \\
\hline 1 & $37(10.5)$ & $4(2.9)$ & \\
\hline 2 & $287(81.8)$ & $102(75.0)$ & \\
\hline 3 & $27(7.7)$ & $30(22.1)$ & \\
\hline ER status & & & 0.077 \\
\hline Negative & $65(18.5)$ & $35(25.7)$ & \\
\hline Positive & $286(81.5)$ & $101(74.3)$ & \\
\hline PR status & & & 0.221 \\
\hline Negative & $94(26.8)$ & $44(32.4)$ & \\
\hline Positive & $257(73.2)$ & $92(67.6)$ & \\
\hline Her-2 status & & & 0.011 \\
\hline Negative & $320(91.2)$ & $113(98.1)$ & \\
\hline Positive & $31(8.8)$ & $23(16.9)$ & \\
\hline Ki-67 status & & & $<0.001$ \\
\hline$\leq 20$ & $172(49.0)$ & $65(47.8)$ & \\
\hline$>20$ & $179(51.0)$ & $71(52.2)$ & \\
\hline No. of PL-1-ALN & & & $<0.001$ \\
\hline 1 & $171(48.7)$ & $16(11.8)$ & \\
\hline 2 & 87 (23.9) & $19(14.0)$ & \\
\hline 3 & $35(10.0)$ & $19(14.0)$ & \\
\hline 4 & $29(8.3)$ & $14(10.3)$ & \\
\hline$\geq 5$ & $32(9.1)$ & $68(50.0)$ & \\
\hline Tumor location & & & 0.978 \\
\hline UOQ & $180(51.3)$ & $71(52.2)$ & \\
\hline LOQ & $36(10.3)$ & $12(8.8)$ & \\
\hline LIQ & $25(7.1)$ & $11(8.1)$ & \\
\hline UIQ & $53(15.1)$ & $19(14.0)$ & \\
\hline Central & $57(16.2)$ & $23(16.9)$ & \\
\hline
\end{tabular}


Table 3: Results of multivariate logistic regression testing the association of each variable with L-2-ALNM

\begin{tabular}{|c|c|c|c|c|c|c|c|}
\hline \multirow[t]{2}{*}{ Variables } & \multirow[t]{2}{*}{ Coefficient } & \multirow[t]{2}{*}{ SE. } & \multirow[t]{2}{*}{ Wald value } & \multirow[t]{2}{*}{$P$ value } & \multirow[t]{2}{*}{ OR } & \multicolumn{2}{|c|}{$95 \% \mathrm{CI}$} \\
\hline & & & & & & Lower & Upper \\
\hline \multicolumn{8}{|l|}{ Histological grade } \\
\hline 1 & & & & 0.055 & & & \\
\hline 2 & 0.922 & 0.590 & 2.444 & 0.069 & 2.514 & 0.791 & 7.984 \\
\hline 3 & 1.506 & 0.659 & 5.224 & 0.118 & 4.508 & 1.239 & 16.399 \\
\hline Tumor size & 0.552 & 0.182 & 9.197 & 0.002 & 1.736 & 1.215 & 2.479 \\
\hline Ki-67 status & 0.728 & 0.252 & 8.357 & 0.004 & 2.072 & 1.264 & 3.395 \\
\hline No. of PL-1-ALN & 0.683 & 0.079 & 74.779 & $<0.001$ & 1.979 & 1.696 & 2.311 \\
\hline Her-2 status & 0.017 & 0.361 & 0.002 & 0.961 & 1.018 & 0.502 & 2.063 \\
\hline Constant & -5.314 & 0.723 & 54.039 & $<0.001$ & 0.005 & & \\
\hline
\end{tabular}

Points

$\begin{array}{lllllllllll}0 & 10 & 20 & 30 & 40 & 50 & 60 & 70 & 80 & 90 & 100\end{array}$

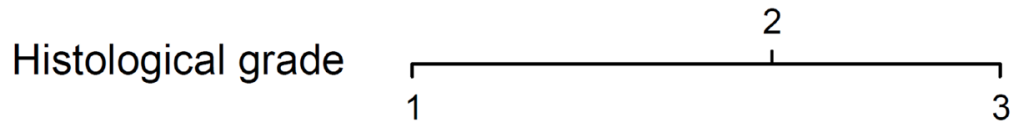

Tumor size

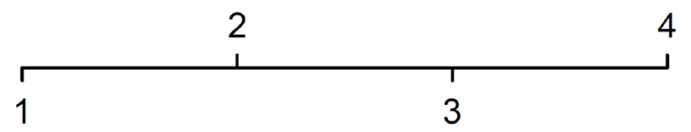

Ki67
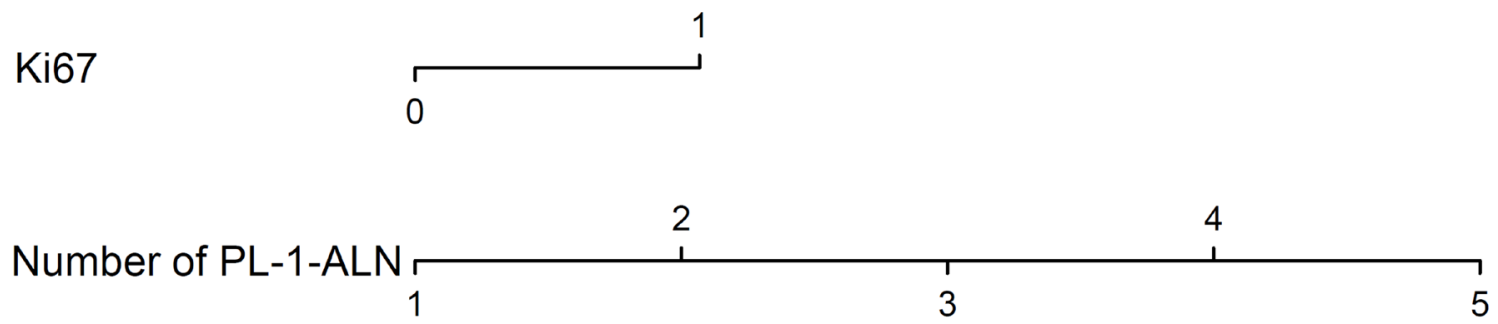

Total Points

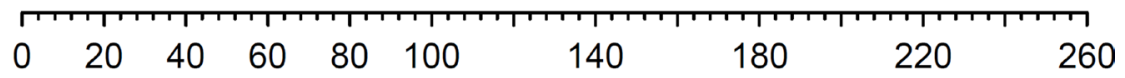

Risk

\begin{tabular}{llllllllllllll}
\hline 0.05 & 0.1 & 0.2 & 0.3 & 0.4 & 0.5 & 0.6 & 0.7 & 0.8 & 0.9 & 0.95
\end{tabular}

Figure 1: Nomogram for predicting the probability of L-2-ALNM. The nomogram has seven rows. The first row is the point assignment for each variable. For each individual patient, each variable is assigned a point value in accordance with the clinicopathological characteristics by delineating a vertical line between the exact variable value and the points line. Thereafter, the Total Points (row 6) can be obtained by summing all of the assigned points for the four variables. Finally, the predictive probability of axillary metastasis can be acquired by drawing a vertical line between Total Points and Risk (the final row). 
Table 4: Predictive values of the L-2-ALNM nomogram at different cutoff values in the validation group

\begin{tabular}{lcccc}
\hline Cut-off values(\%) & $\begin{array}{c}\text { No. of patients and } \\
\text { percentage (\%) }\end{array}$ & No. of falsenegative patients & False-negative rate (\%) & $\begin{array}{c}\text { Negative } \\
\text { predictive } \\
\text { value (\%) }\end{array}$ \\
\hline$<6$ & $55(11.29)$ & 1 & 1.82 & 98.18 \\
$<10$ & $135(27.72)$ & 10 & 7.41 & 92.59 \\
$<15$ & $243(49.90)$ & 25 & 10.29 & 89.71 \\
\hline
\end{tabular}

the predictive values between the modeling and validation groups were similar, which was conducive for further evaluation of the clinical usefulness of the model.

\section{DISCUSSION}

Axillary lymph node status is a significant prognostic factor in patients with primary breast cancer and may plays a decisive role in deciding the therapeutic options [14-16]. With more in-depth research on breast cancer, some researchers believe that patients without axillary lymph node metastasis will not benefit from
ALND [17]. According to a review by Hiken, surgical treatment of breast cancer patients should be divided into three groups [18]: (1) patients without clinically suspected axillary lymph node metastasis for whom axillary lymph node surgery can be completely avoided; (2) patients with limited positive nodes received only SLNB; and (3) patients with more positive axillary lymph nodes must perform ALND [18-19]. Although sentinel lymph node biopsy could be performed in patients with one or two metastatic lymph nodes, it could not be a reasonable treatment for patients with positive axillary lymph nodes confirmed preoperatively by clinical and imaging

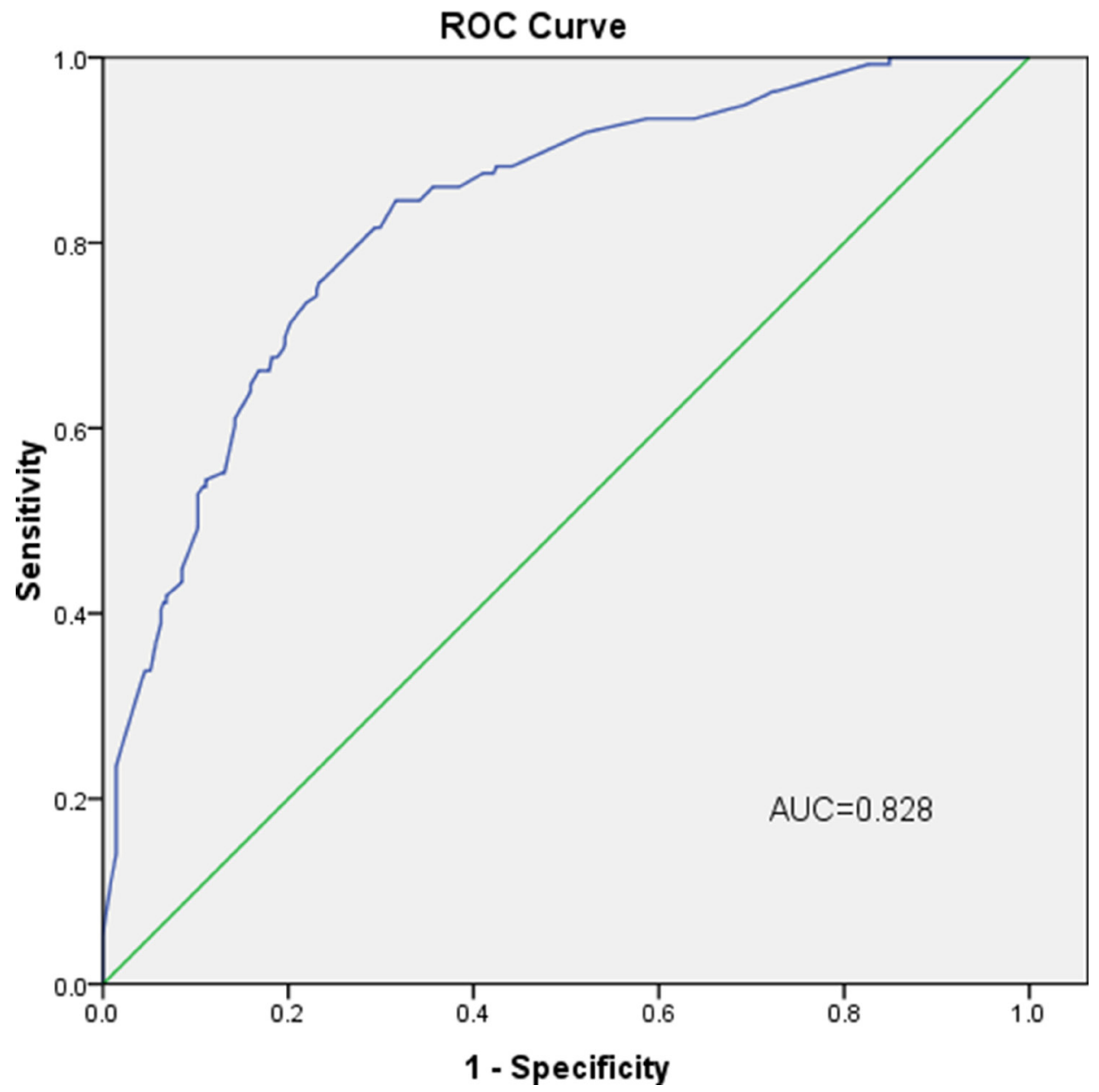

Figure 2: Internal validation using a ROC curve. The AUC value is 0.828 (95\% CI: $0.788-0.868$ ). 


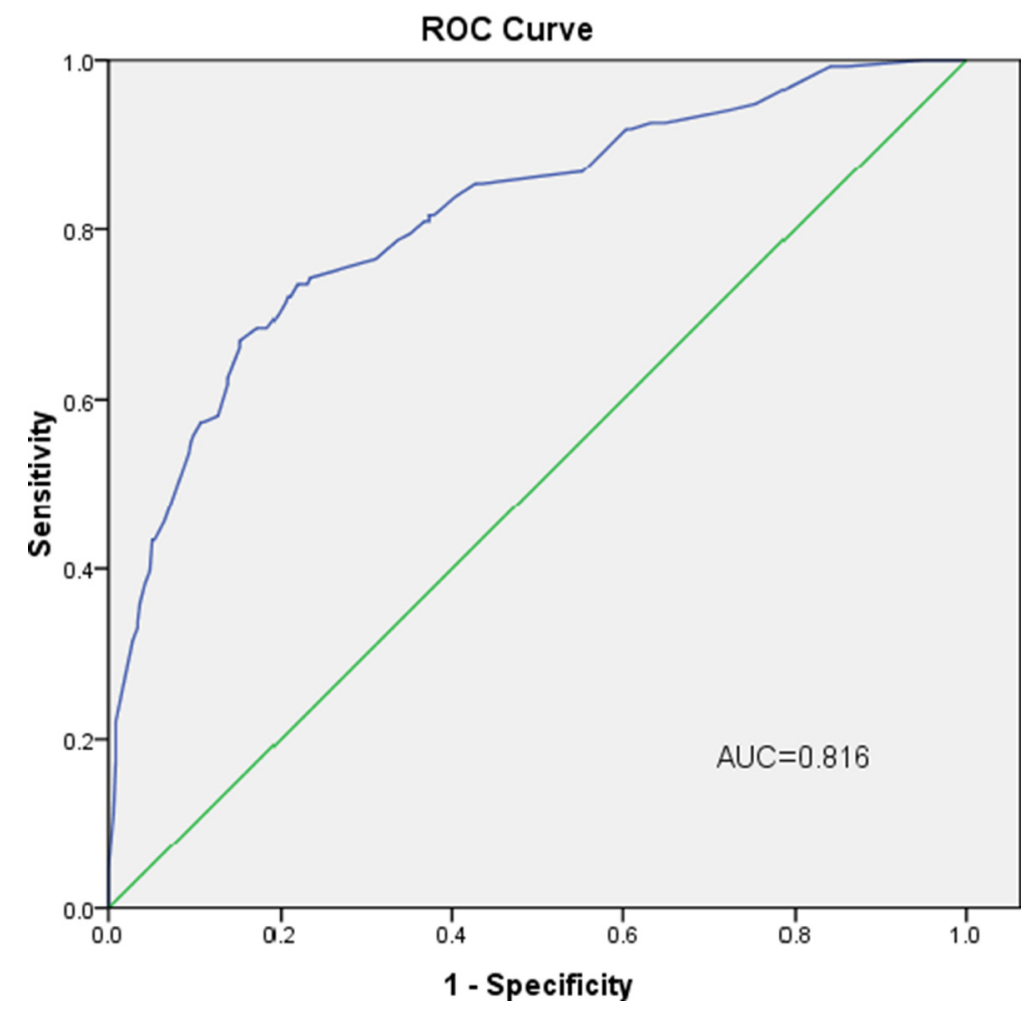

Figure 3: External validation using a ROC curve. The AUC value is 0.816 (95\% CI: $0.772-0.859$ ).

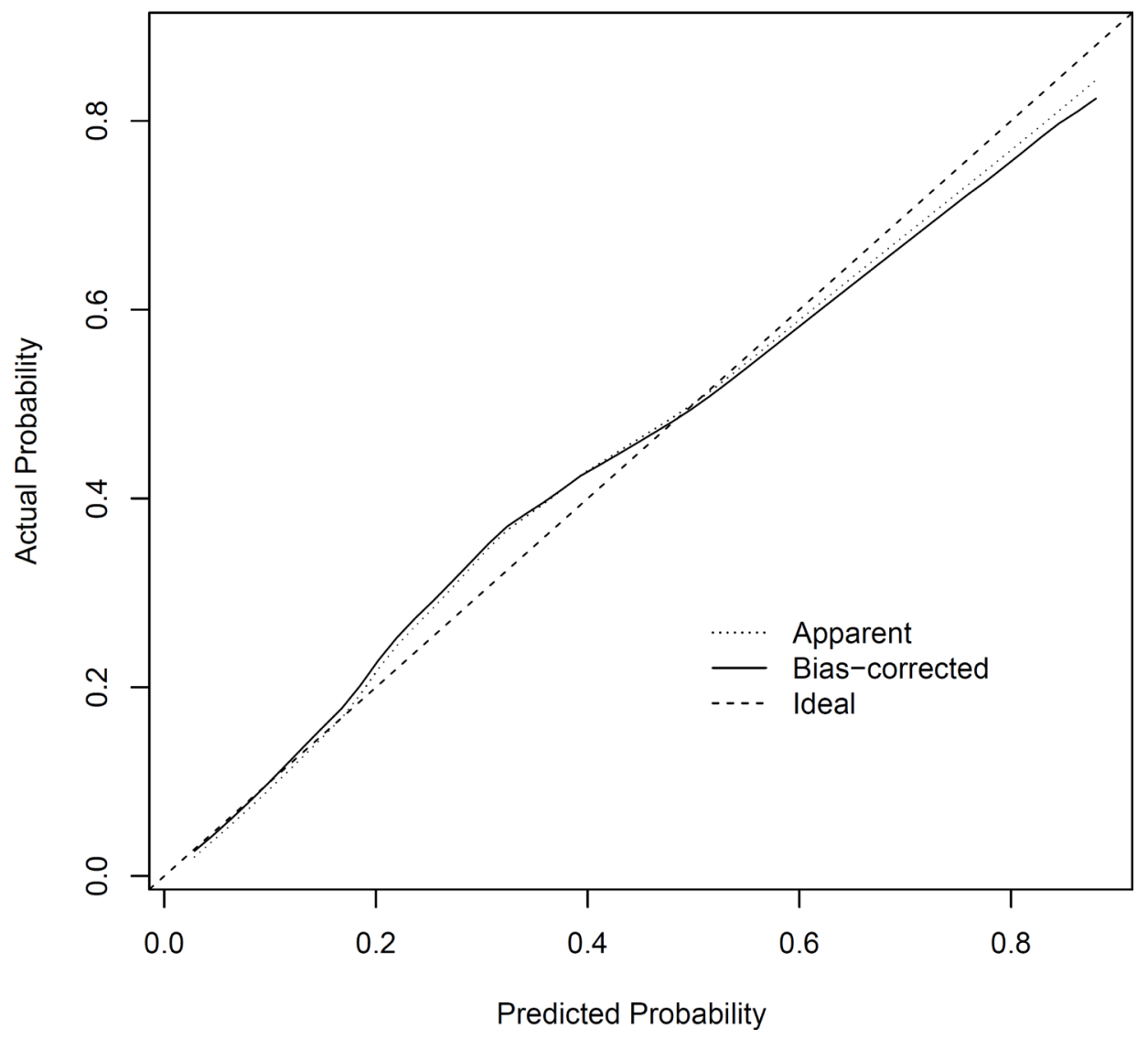

Figure 4: Calibration plot of the nomogram for the probability of L-2-ALNM. 
examinations. And the National Comprehensive Cancer Network (NCCN) guidelines showed that level 1/2 lymph node dissection were performed for the clinical stage I and stage II breast cancer patients with clinically positive nodes. So, our model could be more suitable for these patients with positive axillary lymph nodes confirmed preoperatively by clinical and imaging examinations. In addition, the treatment of omitting axillary lymph node dissection has not been practiced for the patients with one or two sentinel lymph nodes in China. Furthermore some studies showed that the incidence of postoperative complications such as upper arm lymphedema and upper limb numbness was remarkably lower in the patients receiving less lymph nodes dissection compared with patients who have more lymph nodes dissected. $[8,10]$ Therefore, level 1 ALND is a better option for patients with positive level 1 axillary lymph nodes but without L-2-ALNM. Therefore, how to use a noninvasive and reliable tool to accurately screen out these patients without L-2-ALNM become a crucial problem. In the era of individually and precisely tailored treatment, although accurate identification of patients without L-2ALNM is still a challenging problem, predicting model of L-2-ALNM will become increasingly indispensable in formulating surgical regimens.

The model to predict L-2-ALNM in patients with L-1-ALNM has not previously been reported. In the present study, we developed a nomogram consisting of four independent factors relevant to L-2-ALNM in patients with L-1-ALNM. With the help of the model, an innovative approach of deciding to omit L-2-ALND offers several advantages compared with ALND. First, the elimination of unnecessary L-2-ALND can provide patients with better postoperative quality of life. Second, the model has the advantage of reducing the patient's medical expenses, such as reducing the cost of additional surgery, some diagnostic fees and lymph node pathology fees.

Predicting L-2-ALNM in level 1 node-positive breast cancer patients is critical to understand the prognosis of the patients, which directly determines the treatment of level 2 axillary lymph node. We retrospectively analyzed the clinical and pathological data of 974 patients with breast cancer, and established a predictive model to assess the risk of L-2-ALNM. With the help of the univariate and multivariate analyses, four independent predictors associated with L-2-ALNM were contained in the prediction model. They were tumor size, Ki-67 status, HER-2 status, histological grade, and number of PL-1-ALN. Many other studies have reported that tumor size and histological grade are risk factors for axillary lymph node metastasis [8, 20-22], consistent with the results we have studied. Ki-67 status as a predictor of axillary lymph node metastasis was reported by some researchers [23]. The AUC value of our modeling group was 0.828 . We then applied an additional set of independent data to validate the model and gained an AUC value of 0.816 , which indicated it was a model with excellent discrimination ability.

To the best of our knowledge, this is the first nomogram prediction model of level 2 axillary lymph node status in breast cancer patients with level 1 axillary lymph node metastasis reported in the English literature. In the light of the results, we believe that the proposed L-2-ALNM nomogram is both valid and useful in Chinese populations.

To further evaluate the clinical usefulness of the L-2ALNM prediction model, we calculated the false-negative value of our model at probability cutoff values of $<6 \%$, $<10 \%$, and $<15 \%$ in the validation groups (Table 4 ). During the calculation, we observed that only the populations with a low risk of metastasis were exempted from undergoing L-2-ALND.

The study has reported that the overall falsenegative rate of SLNB was $8.4 \%(0-29 \%)$ [24]. Therefore, we assume that most surgeons can accept a false-negative rate of $0-8 \%$. In the validation group, when the cutoff values were $<6 \%$ and $<10 \%$, the false-negative rates were $1.82 \%$ and $7.41 \%$, respectively, which were lower than the false-negative rate of sentinel lymph node biopsy. The proportions of patients with L-2-ALNM rate of $<6 \%$ was $11.29 \%$ in the validation group. Meanwhile, the proportion of the patients with L-2-ALNM rate of $<10 \%$ was $27.72 \%$ in the validation group. This finding suggests that our prediction model could achieve the desired predictive effects. Hence, based on our prediction model, if the L-2-ALNM rates are $<6 \%$ or $<10 \%$, these patients could be exempted from L-2-ALND based on the actual situation. This is particularly so for elderly patients with comorbidities, as ALND will not only increase the risk of surgery but also cause serious postoperative complications.

The methods of breast cancer treatment are continuously changing, and the management of axillary lymph nodes is also continuously evolving. As clinical research improves, the prediction of residual axillary lymph nodes will become increasingly important, as this will directly affect the development of treatment protocols for axillary lymph nodes. Our prediction model includes 4 common clinicopathological variables that can be obtained through routine examinations. Our predictive model had preferable predictive ability and clinical utility. As demonstrated in this study, the stability of our model allowed us to believe that the model was universally applicable to other Asian patient populations, although further validation in other populations is also desirable.

Apparently, the results of prediction models are more reliable than clinical speculation. Even though our prediction model shows better predictive power, but it still has limitations that need to be addressed. First, our model was developed based on a retrospective, single-center study and thus requires further validation in other research 
settings. In addition, only the patients with invasive ductal carcinoma were included in our study, which decreased the scope of application of the model.

In conclusion, we established an accurate, objective, and easy-to-use model to predict the probability of L-2ALNM in the breast cancer patients with L-1-ALNM. In virtue of our nomogram, the surgeons could consider avoiding their L-2-ALND for low-risk patients with L-2ALNM.

\section{MATERIALS AND METHODS}

\section{Patients}

Data were collected from 3444 breast cancer patients diagnosed with invasive ductal carcinoma who were treated between January 2010 and December 2014 at the Liaoning Cancer Hospital and Institute. Among them, a total of $34.2 \%$ (1179) patients had positive level 1 axillary lymph nodes. After excluding 205 patients with incomplete relevant information, a total of 974 patients were included in the study. Clinical data included in the analysis were the following: data on tumor size (categorized as $\mathrm{T} 1 \leq 2 \mathrm{~cm}, 2 \mathrm{~cm}<\mathrm{T} 2 \leq 3 \mathrm{~cm}, 3 \mathrm{~cm}<\mathrm{T} 3$ $\leq 5 \mathrm{~cm}, \mathrm{~T} 4>5 \mathrm{~cm}$ ), tumor location, immunohistochemistry (IHC) (estrogen receptor [ER], progesterone receptor [PR], human epidermal growth factor receptor 2 [Her-2], Ki-67), histological grade, age, menopausal status, and number of positive level 1 axillary lymph nodes (PL-1ALN).

The inclusion criteria were as follows: 1) diagnosed with invasive ductal carcinoma, 2) operable primary breast cancer confirmed by core biopsy or open biopsy, 3) had positive level 1 axillary lymph nodes confirmed by pathology postoperatively, 4) underwent both radical excision of the primary tumor and ALND, 5) informed consent was obtained. Exclusion criteria were as follows: missing data, negative level 1 axillary lymph node confirmation by pathology, skin invasion, distant metastatic disease at diagnosis, not invasive ductal carcinoma, and receipt of neoadjuvant chemotherapy. The eligible patients were randomized $1: 1$ and divided into a modeling set (nomogram construction) and a validation set (nomogram validation). This study was reviewed and approved by the Ethics Committee of Liaoning Cancer Hospital and Institute.

\section{Treatment}

Surgical treatment was performed on the basis of the Chinese breast cancer guidelines. Surgery included resection of the primary tumor and performance of level 1 and level 2 ALND. The histological status and quantity of level 1 and level 2 nodes were analyzed retrospectively.

\section{Data extraction}

We utilized each of the following variables: tumor characteristics (histological grade; clinical tumor size; tumor location; and ER, PR, and HER-2 status; and Ki-67 status), age, menopausal status, and nodal status including level 1 and level 2 from surgical pathology reports after ALND.

\section{Pathologic evaluation}

The Chinese breast cancer guidelines were used to evaluate surgical specimens. Tumors with $\geq 10 \%$ nuclear-stained cells were considered positive for ER and PR. Ki67 expression $\geq 20 \%$ was considered positive. HER-2 positivity was defined as a score of $3+$ on IHC or amplification on FISH [13]. If a pathologist scored the IHC 2+, the HER-2 status was further investigated by FISH. In addition, the grade of breast cancer was determined by the Nottingham Histologic Score system.

\section{Statistical analysis}

The chi-square test or Fisher's exact were utilized for the categorical data, and descriptive statistics were used for the within-group or between-group comparisons of independent samples. All statistical analyses were performed using SPSS 17.0 statistical software (SPSS Inc., Chicago, IL, USA) and R software (version 3.1.0). A two-tailed $P$ value $<0.05$ was considered statistically significant. All reported $P$ values are two-sided.

Univariate analysis of the 10 factors described above was performed to decide which one was associated with L-2-ALNM. Variables that were statistically significant $(P<0.05)$ in the univariate logistic analysis in the modeling group were included in the multivariate logistic regression analysis, which was used to screen independent predictors for L-2-ALNM. Independent predictors $(P<0.05$ in the multivariate logistic regression analysis $)$ were used to construct a well-calibrated nomogram for predicting the probability of L-2-ALNM. An additional set of 487 Chinese patients in the validation group was used to validate the predictive model.

The performance of the model was quantified with respect to discrimination and calibration. First, discrimination is the predictor's ability to separate patients with different responses or events. The ROC curve was drawn, and the AUC was used to assess the discriminatory abilities of the model. Second, calibration is the agreement between the frequencies of observed outcomes and the probabilities predicted by the model. The calibration of the nomogram was performed internally by a calibration plot with bootstrap sampling $(n=1000)$. The nomogram was defined as wellcalibrated if the calibration plot of the model fell along 
the 45-degree line. The Hosmer-Lemeshow test and visualize inspection of the plots were used to evaluate the calibration plot, and $P>0.05$ indicated a good fit.

\section{Abbreviations}

ALND: axillary lymph node dissection; L-2-ALNM: level 2 axillary lymph node metastasis; L-1-ALNM: level 1 axillary lymph node metastasis; PL-1-ALN: positive level 1 axillary lymph nodes; ROC: receiver operating characteristic; AUC: areas under the receiver operating characteristic curve: L-2-ALND: level 2 axillary lymph node dissection; ORs: odds ratios; CIs: confidence intervals; UOQ, upper outer quadrant; UIQ, upper inner quadrant; LOQ, lower outer quadrant; LIQ, lower inner quadrant.

\section{Author contributions}

Yanlin Jiang and Caigang Liu participated in the design of the study. Yanlin Jiang and Hao Zhang performed the statistical analysis and drafted the manuscript. CaigangLiu, HongXu, and Hao Zhang participated in the coordination of the study. All authors read and approved the final manuscript.

\section{ACKNOWLEDGMENTS}

This work was supported in part by China Postdoctoral Science Foundation (2015M570259) and the China National Natural Science Foundation (31601142, 81402384, and 81572609).

\section{CONFLICTS OF INTEREST}

No competing interests declared.

\section{REFERENCES}

1. Jin X, Jiang YZ, Chen S, Shao ZM, Di GH. A Nomogram for Predicting the Pathological Response of Axillary Lymph Node Metastasis in Breast Cancer Patients. Sci Rep. 2016; 6:32585

2. DeSantis C, Siegel R, Bandi P, Jemal A. Breast cancer statistics, 2011. CA Cancer J Clin. 2011; 61:409-418.

3. Jemal A, Bray F, Center MM, Ferlay J, Ward E, Forman D. Global cancer statistics. CA Cancer J Clin. 2011; 61:69-90.

4. Qiu SQ, Zeng HC, Zhang F, Chen C, Huang WH, Pleijhuis RG, Wu JD, van Dam GM, Zhang GJ. A nomogram to predict the probability of axillary lymph node metastasis in early breast cancer patients with positive axillary ultrasound. Sci Rep. 2016; 6:21196.

5. Rattay T, Muttalib M, Khalifa E, Duncan A, Parker SJ. Clinical utility of routine pre-operative axillary ultrasound and fine needle aspiration cytology in patient selection for sentinel lymph node biopsy. Breast. 2012; 21:210-214.
6. Velanovich V, Szymanski W. Quality of life of breast cancer patients with lymphedema. Am J Surg. 1999; 177:184-187; discussion 188.

7. Giuliano AE, Hunt KK, Ballman KV, Beitsch $\mathrm{PD}$, Whitworth PW, Blumencranz PW, Leitch AM, Saha S, McCall LM, Morrow M. Axillary dissection vs no axillary dissection in women with invasive breast cancer and sentinel node metastasis: a randomized clinical trial. JAMA. 2011; 305:569-575.

8. Schipper RJ, Moossdorff M, Nelemans PJ, Nieuwenhuijzen GA, de Vries B, Strobbe LJ, Roumen RM, van den Berkmortel F, Tjan-Heijnen VC, Beets-Tan RG, Lobbes MB, Smidt ML. A model to predict pathologic complete response of axillary lymph nodes to neoadjuvant chemo(immuno)therapy in patients with clinically node-positive breast cancer. Clin Breast Cancer. 2014; 14:315-322.

9. Jung SY, Shin KH, Kim M, Chung SH, Lee S, Kang HS, Lee ES, Kwon Y, Lee KS, Park IH, Ro J. Treatment factors affecting breast cancer-related lymphedema after systemic chemotherapy and radiotherapy in stage II/III breast cancer patients. Breast Cancer Res Treat. 2014; 148:91-98.

10. Ashikaga T, Krag DN, Land SR, Julian TB, Anderson SJ, Brown AM, Skelly JM, Harlow SP, Weaver DL, Mamounas EP, Costantino JP, Wolmark N. Morbidity results from the NSABP B-32 trial comparing sentinel lymph node dissection versus axillary dissection. J Surg Oncol. 2010; 102:111-118.

11. Park HS, Kim HY, Park S, Kim EK, Kim SI, Park BW. A nomogram for predicting underestimation of invasiveness in ductal carcinoma in situ diagnosed by preoperative needle biopsy. Breast. 2013; 22:869-873.

12. Van Zee KJ, Manasseh DM, Bevilacqua JL, Boolbol SK, Fey JV, Tan LK, Borgen PI, Cody HS 3rd, Kattan MW. A nomogram for predicting the likelihood of additional nodal metastases in breast cancer patients with a positive sentinel node biopsy. Ann Surg Oncol. 2003; 10:1140-1151.

13. Wolff AC, Hammond ME, Hayes DF. Re: predictability of adjuvant trastuzumab benefit in N9831 patients using the ASCO/CAP HER2-positivity criteria. J Natl Cancer Inst. 2012; 104:957-958.

14. Carter CL, Allen C, Henson DE. Relation of tumor size, lymph node status, and survival in 24,740 breast cancer cases. Cancer. 1989; 63:181-187.

15. Rosen PP, Groshen S, Saigo PE, Kinne DW, Hellman S. Pathological prognostic factors in stage I (T1N0M0) and stage II (T1N1M0) breast carcinoma: a study of 644 patients with median follow-up of 18 years. J Clin Oncol. 1989; 7:1239-1251.

16. Quiet CA, Ferguson DJ, Weichselbaum RR, Hellman S. Natural history of node-negative breast cancer: a study of 826 patients with long-term follow-up. J Clin Oncol. 1995; 13:1144-1151.

17. Fein DA, Fowble BL, Hanlon AL, Hooks MA, Hoffman JP, Sigurdson ER, Jardines LA, Eisenberg BL. Identification of women with T1-T2 breast cancer at low risk of positive axillary nodes. J Surg Oncol. 1997; 65:34-39. 
18. Hieken TJ. The promise of axillary imaging in individualized surgical management of breast cancer patients: another step forward. Ann Surg Oncol. 2014; 21:3369-3371.

19. Gentilini O, Veronesi U. Abandoning sentinel lymph node biopsy in early breast cancer? A new trial in progress at the European Institute of Oncology of Milan (SOUND: Sentinel node vs Observation after axillary UltraSouND). Breast. 2012; 21:678-681.

20. Bevilacqua JL, Kattan MW, Fey JV, Cody HS 3rd, Borgen PI, Van Zee KJ. Doctor, what are my chances of having a positive sentinel node? A validated nomogram for risk estimation. J Clin Oncol. 2007; 25:3670-3679.

21. Viale G, Zurrida S, Maiorano E, Mazzarol G, Pruneri G, Paganelli G, Maisonneuve P, Veronesi U. Predicting the status of axillary sentinel lymph nodes in 4351 patients with invasive breast carcinoma treated in a single institution. Cancer. 2005; 103:492-500.
22. Klar M, Foeldi M, Markert S, Gitsch G, Stickeler E, Watermann D. Good prediction of the likelihood for sentinel lymph node metastasis by using the MSKCC nomogram in a German breast cancer population. Ann Surg Oncol. 2009; 16:1136-1142.

23. Kilickap S, Kaya Y, Yucel B, Tuncer E, Babacan NA, Elagoz S. Higher Ki67 expression is associates with unfavorable prognostic factors and shorter survival in breast cancer. Asian Pac J Cancer Prev. 2014; 15:1381-1385.

24. Lyman GH, Giuliano AE, Somerfield MR, Benson AB 3rd, Bodurka DC, Burstein HJ, Cochran AJ, Cody HS 3rd, Edge SB, Galper S, Hayman JA, Kim TY, Perkins CL, et al. American Society of Clinical Oncology guideline recommendations for sentinel lymph node biopsy in earlystage breast cancer. J Clin Oncol. 2005; 23:7703-7720. 